\title{
Computational Intelligence for Smart Environments
}

\author{
Macarena Espinilla ${ }^{1}$ and Chris Nugent ${ }^{2}$ \\ ${ }^{1}$ Department of Computer Science, University of Jaén, \\ Campus Las Lagunillas \\ Jaén, 23071, Spain \\ E-mail:mestevez@ujaen.es \\ ${ }^{2}$ School of Computing and Mathematics, Ulster University, \\ Jordanstown campus, \\ Northern Ireland, BT37 0QB, UK \\ E-mail:cd.nugent@ulster.ac.uk
}

Received 26 June 2017

Accepted 8 July 2017

\section{Preface}

Smart environments are a multi-disciplinary research area within which technology is pervasively and ubiquitously deployed to ensure that actions are supported in an appropriate and sensitive manner. Such a solution is possible thanks to the mechanisms which support the aggregation and fusion of data along with intelligent data analytics techniques to offer appropriate decisions based on the data gleaned from the environment. In this context, the core approaches of computational intelligence (Fuzzy Logic, Neural Networks, Evolutionary Computation and Probabilistic Reasoning) are excellent tools to be employed in smarts environments.

This special issue, produced following the 10th International Conference on Ubiquitous Computing \& Ambient Intelligence (UCAmI 2016), held in Canary Islands, (Spain) from November, 29th to December 2nd, 2016 is a culmination of submissions to an open call for papers with a focus on recent advancements within smart environments through the usage of computational intelligence techniques.

This special issue comprises seven articles which were submitted to the call for papers and accepted following peer review. Each of the articles presents advances in research in smart environments applied to arrange of interesting and relevant application domains. We hope that this special issue provides an inspirational collection of ideas, techniques, and methodologies for smart environments through use of computational intelligence techniques which will continue to stimulate further research within this state-of-the-art domain.

In the first paper, "Particle swarm optimization and harmony search based clustering and routing in wireless sensor networks" by Veena Anand and Sudhakar Pandey, an approach for improving network lifetime by using particle swarm optimization based clustering and harmony search based routing in wireless sensor networks is described.

Similarly, in the context of the wireless sensor networks, Sridevi Ponmalar P. and Jawahar Senthil Kumar V. present the paper entitled "Hybrid firefly variants algorithm for localization optimization in wireless sensor network". In this paper, bio-inspired hybrid algorithms are analyzed, designed and implemented to optimize the localization error in wireless sensor networks.

The paper entitled "Classifier optimized for resource-constrained pervasive systems and energyefficiency" is proposed by Niklas Karvonen, Lara Lorna Jimenez, Miguel Gomez Simon, Joakim Nilsson, Josef 
Hallberg and Basel Kikhia is the third paper of this special issue. In this paper, a classifier optimized for resource-constrained pervasive systems and energyefficiency referred to as CORPSE is proposed. The solution outlined in the paper has been specifically targeted for implementation in Field-Programmable Gate Arrays. The proposed solution has a simplistic design and has provided performance levels similar to a range of commonly used machine learning algorithms.

Caglar Uyulan and Turker Tekin Erguzel propose the paper entitled "Analysis of time - frequency EEG feature extraction methods for mental task classification". This paper provides an interesting and extensive study of methods used for feature extraction of EEG signals which can be used to classify mental tasks in support of a brain computer interface application.

The context of the fifth paper of this special issue is focused on managing situations of multi-occupancy. Vahid Ghasemi and Ali Akbar Pouyan present the paper entitled "Modeling users' data traces in multi-resident ambient assisted living environments". This paper presents a novel method based on Markov chain for modeling users' data traces in multi-resident sensorbased ambient assisted living environments, offering a promising paradigm.

"Energy-efficient acoustic violence detector for smart cities" by Marta Bautista-Durán, Joaquín GarcíaGómez, Roberto Gil-Pita, Inma Mohíno-Herranz, and
Manuel Rosa-Zurera, is the sixth paper of this special issue. This paper proposes the use of genetic algorithms to identify the best subset of features taking into consideration constrained computational costs. The computed results presented in the paper demonstrate the viability of the proposed solution in low cost devices.

The final paper of this special issue entitled "Fuzzylogic-based application to combat gender violence" by José Á. Concepción-Sánchez, Pino Caballero-Gil and Jezabel Molina-Gil presents the use of the fuzzy logic in a system to compute the distance between victim and aggressor in order to avoid false alarms.

As Guest Editors of this special issue, we would like to take this opportunity to thank all the authors for their efforts in the preparation of their manuscripts. In addition, we wish to express our deepest gratitude to the referees who provided very useful and thoughtful feedback and our sincere thanks go to the Editors-inChief for their kind help and support. We hope that you enjoy reading the articles within this Special Issue and that the published work are inspirational in contributing to progression the state-of-the-art in computational intelligence techniques in smart environments.

Finally, the Editors which to acknowledge partial support from the REMIND Project from the European Union's Horizon 2020 research and innovation programme under the Marie Skłodowska-Curie grant agreement No 734355. 